\title{
Corset platysmaplasty - 'The cervical cinch'
}

\author{
Peter G Whidden MD FRCSC FACS \\ Calgary, Alberta
}

The most important visual criteria for defining the youthful neck can be met by the corset platysmaplasty in many facelift patients.

\section{VISUAL CRITERIA FOR THE YOUTHFUL NECK (1)}

1. A well-defined inferior mandibular border.

2. An acute cervicomental angle.

3. Absence of submandibular gland bulges (or ptosis).

4. Absent visible paramedian muscle bands and contour irregularities.

5. Visible but not prominent thyroid bulge (laryngeal prominence).

6. Visible anterior sternocleidomastoid border.

\section{CONTEMPORARY METHODS OF PLATYSMAPLASTY $(2,3)$}

1. Vertical excision of prominent muscle borders (Figure 1).

2. Upper midline plication (Figure 2).

3. Anterior wedge resection below the level of plication (Figure 3).

4. Medial and/or lateral partial-width transection (1).

5. Full-width muscle transection and posterior shift of the lateral muscle (Figure 4).

6. Full-width muscle resection below the level of the hyoid.

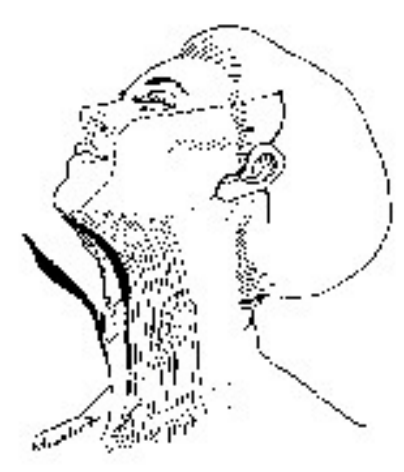

Figure 1) Vertical excision of prominent muscle borders

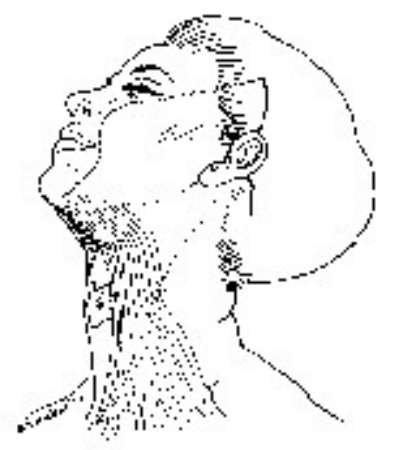

Figure 2) Upper midline plication 


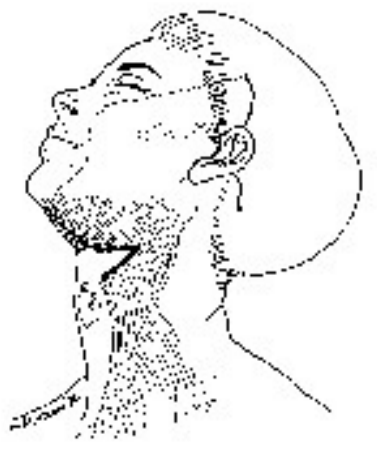

Figure 3) Anterior wedge resection below the level of Figure 4) Full-width muscle transection and plication

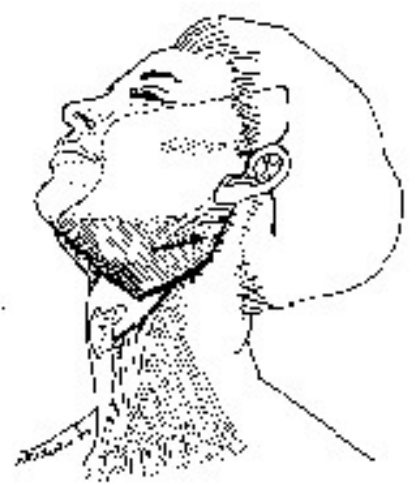

\section{UNDESIRABLE SEQUELAE FOLLOWING CONTEMPORARY PLATYSMAPLASTY (4)}

1. Recurrence of paramedian muscle bands as a result of leaving unjoined muscle edges.

2. Visible submandibular gland bulges as a result of overlying fat removal unmasking the glands.

3. Visible submandibular gland bulges as a result of loss of musculofascial support of the glands.

4. Inadequate subplatysmal fat resection resulting in submental fullness (obtuse cervicomental angle).

5. Submental depression as a result of submental lipectomy with inadequate submental platysma midline imbrication.

6. Skeletonization of the thyroid cartilage.

Figure 5 (left and centre) illustrates some of the problems associated with traditional methods. 

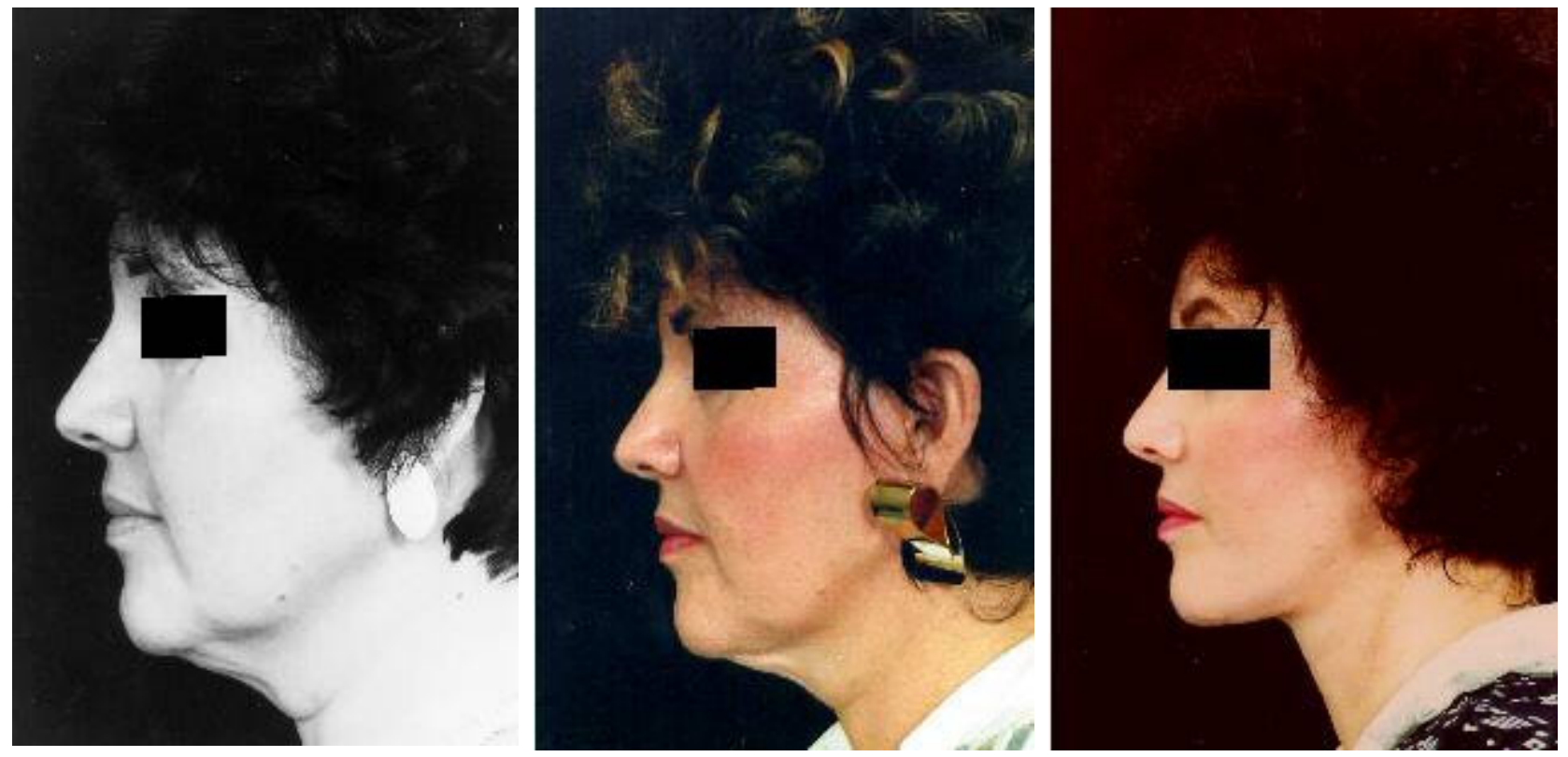

Figure 5) A 39-year-old woman with a fatty neck and flaccid platysma, shown before and three years after a conservative face lift, showing no improvement of the neck, then shown three years after face and neck lift with superficial muscoaponeurotic system advancement, subcutaneous, subplatysmal and prehyoid neck lipectomy, and corset platysmalplasty
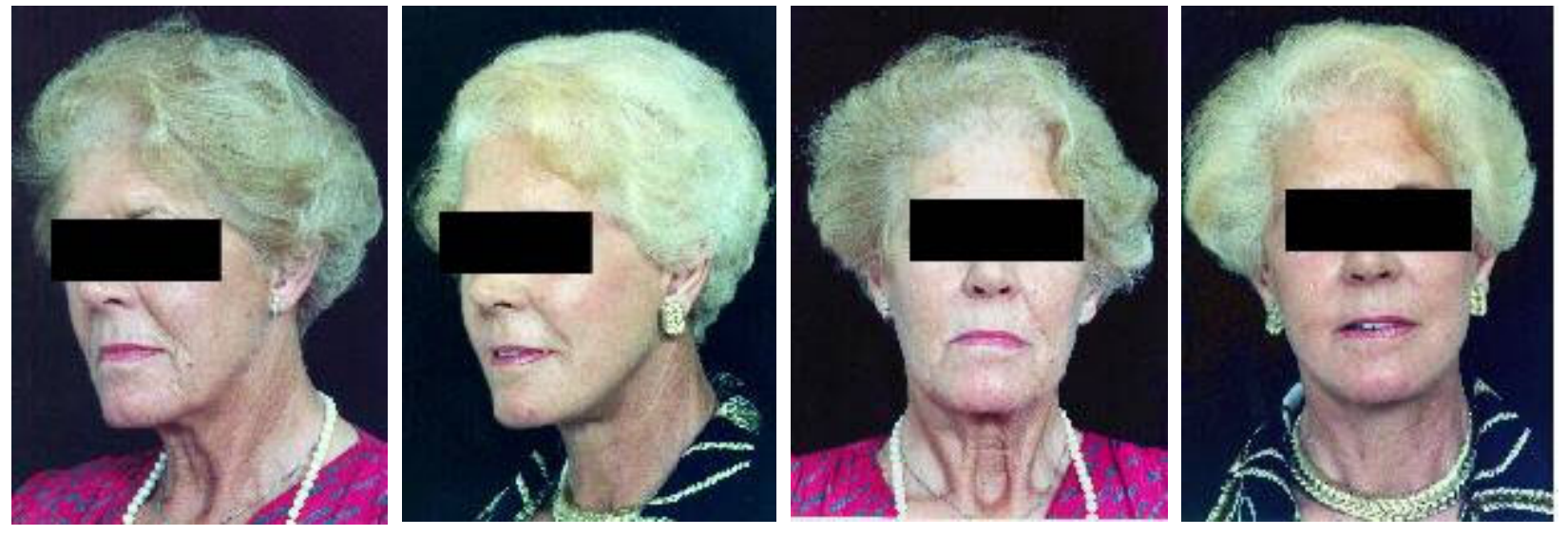

Figure 6) A 62-year-old woman with long prominent paramedian platysma bands is shown before and one year after face and neck lift with superficial muscoaponeuronic system advancement, subplatysmal and prehyoid neck lipectomy and corset platysmaplasty 

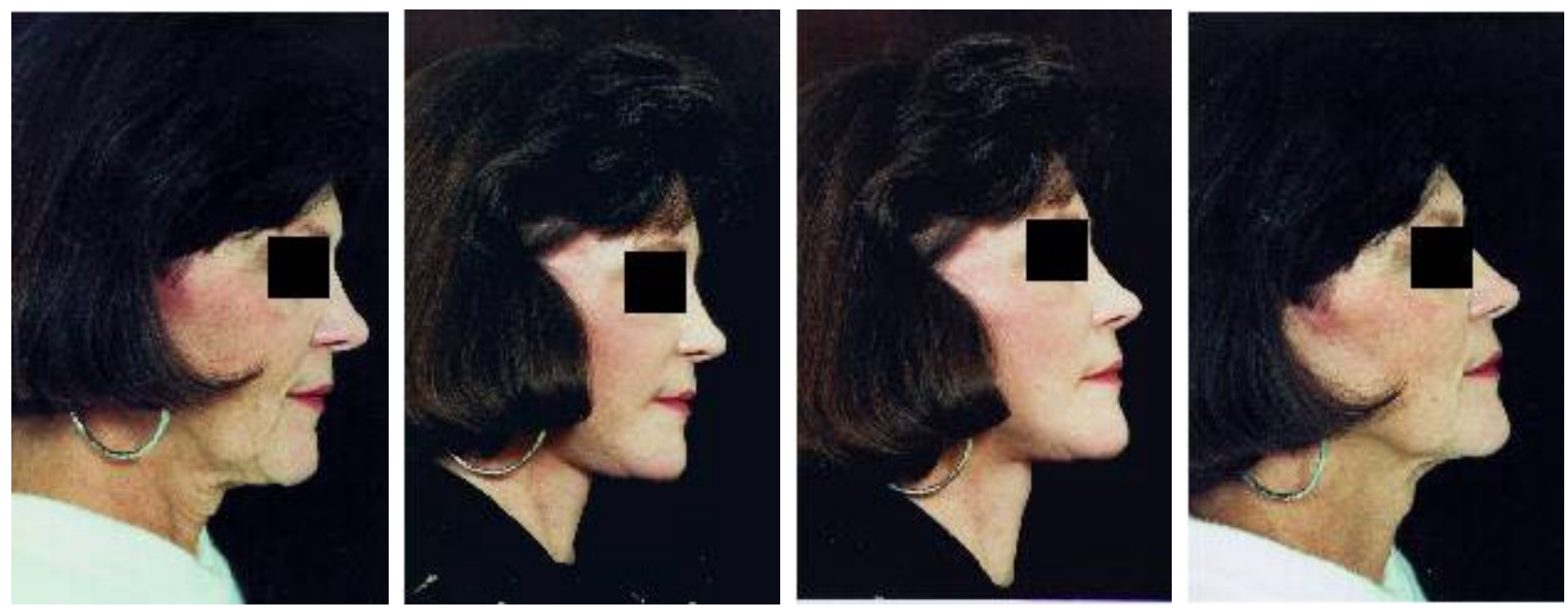

Figure 7) A 54-year-old woman with flaccid platysma shown before and one year after coronal lift, four-lid blepharoplasty, face and neck lift with superficial muscoaponeurotic system advancement, prehyoid lipectomy and corset platysmaplasty

\section{OBJECTIVES OF PLATYSMAPLASTY}

The ideal platysmaplasty should:

1. Allow controlled neck contouring under direct visualization.

2. Allow appropriate subplatysmal and prehyoid lipectomy.

3. Not require muscle transection or resection.

4. Strengthen musculofascial support correcting submandibular gland ptosis (5).

5. Create a continuous sheet of muscle devoid of bands or bunching of retracted muscle fibres and covering the thyroid cartilage completely (5).

6. Facilitate skin redraping and closure without tension or conspicuous cutaneous scars.

When we first began to join the upper platsyma borders together in the midline (Figure 2), we observed that the unjoined medial borders below appeared to become more prominent, and logically we carried out either an anterior wedge resection (Figure 3) or a full-width muscle transection (Figure 4) on either side below the level of plication to "break the bowstrung bands and to allow the cut muscle edges to settle back into the hollow of the neck" (5). However, the meticulous multilayered, full height medial plastysma border advancement and imbrication (corset platysmaplasty) will often achieve a smoother and more youthful looking neck contour without cutting platysma.

\section{SURGICAL TECHNIQUE}

Through a $4 \mathrm{~cm}$ long submental incision, subcutaneous dissection is carried to within two fingerbreadths of the suprasternal notch anteriorly and over the bodies of 
sternocleidomastoid muscle laterally and over the horizontal ramus of the mandible and the buccal region of the cheek superiorly (Figure 8).

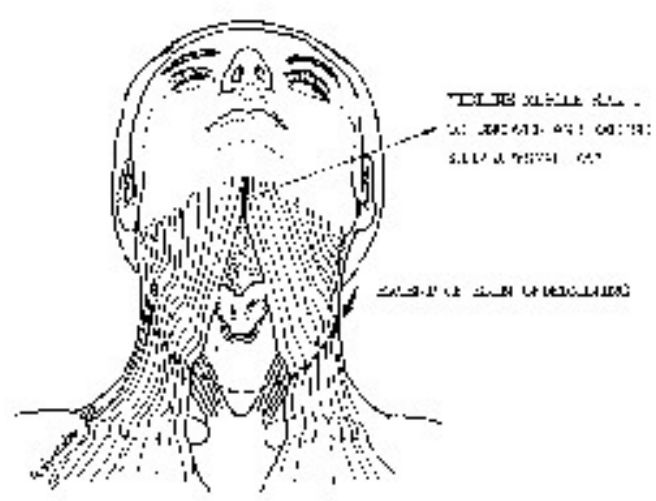

Figure 8) After a meticulous removal of preplatysmal fat, the medial borders of the platysma are separated to remove subplatysmal and prehyoid fat

[Text in figure: Midline muscle split to uncover and excise subplatysmal fat / Extent of skin undermining]]

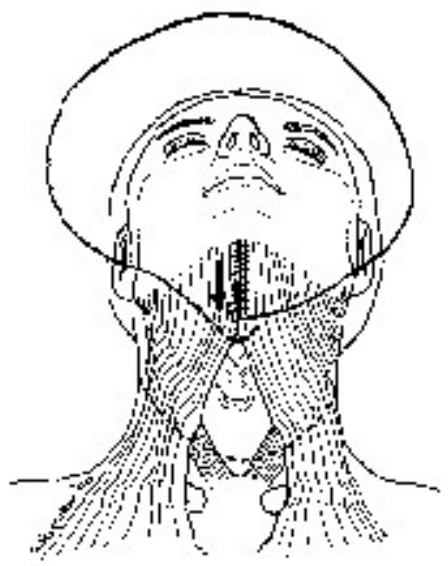

Figure 9) A continuous suture of 3-0 Dexon or Vicryl begins at the mentum and joins the medial borders of platysma together and includes a bite of the anterior periosteum of the hyoid

With the coagulation current, dissection is carried down to the medial borders of platysma and laterally to the sternocleidomastoid, removing a sheet of anterior cervical and submental fat en bloc (of variable breadth and thickness). The medial borders of platysma are then gently raised and defined with the coagulation current, and subplatysmal dissection is carried as far laterally as the submandibular glands, taking care not to 'buttonhole' this thin muscle. Excess midline subplatysmal fat is then removed from the fascia overlying mylohyoid and anterior bellies of digastric, and the deep cervical fascia from the hyoid bone to the thyroid cartilage and cricoid cartilage, skeletonizing the hyoid and larynx (it may be necessary to split the platysma decussation to gain access to the submental subplatysmal fat) (Figure 8).

The full-height midline plication of the medial platysmal borders begins at the mentum (Figure 9) and continues as a running 3-0 polyglycolic acid simple suture to a point beyond the thyroid cartilage, within $3 \mathrm{~cm}$ of the suprasternal notch, then reverses direction (Figure 10) and continues cephalad, imbricating the first row of suture with a smoother, tighter second row concluding at the mentum, only to reverse direction (Figure 11) and imbricate this second row to form a third seam. Finally, this long running suture is tied precisely at the hyoid level. (A continuous suture produces a smoother suture line, distributes tension evenly, and is faster in execution.) 


\section{Corset platysmaplasty}

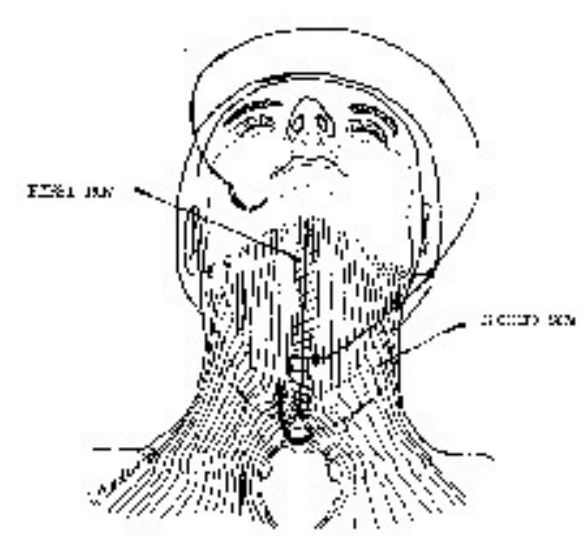

Figure 10) This suture joins the medial borders of the platysma to within two fingerbreadths of the suprasternal notch then reverses direction, taking small bites outside the first row of sutures and advancing platysma muscle medially to form a smooth midline seam, running cephalad toward the mentum

[Text in figure: First row / Second Row]

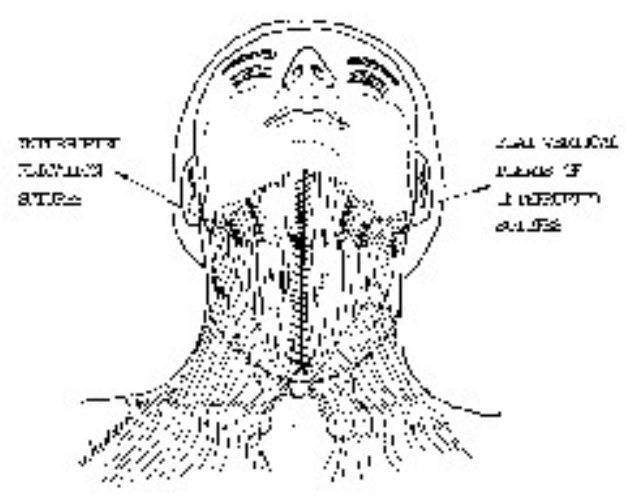

Figure 12) Short flat vertical muscle pleats of interrupted sutures are placed to minimize the bulging of the submandibular salivary glands producing the completed platysmal muscle corset (5)

[Text in figure: Interrupted plication sutures / Flat vertical pleats of interrupted sutures]]

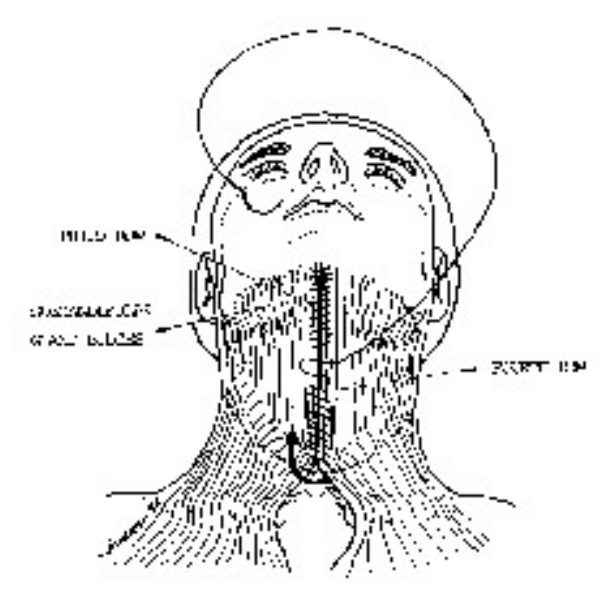

Figure 11) At this point, the same suture reverses direction and proceeds caudad, taking bites just outside the second row of running sutures, further smoothing the submental platysmal sheet. It is then finally knotted over the hyoid

[Text in figure: Third row / Submandibular gland bulges / Fourth row] 
The platysma often remains loose beneath the submandibular glands and a vertical muscle pleat of running or interrupted imbricating sutures flattens the submandibular gland bulges and fortifies their support (Figure 12).

The rhytidectomy flaps are then raised on each side, dissecting to the nasolabial crease and along the horizontal ramus of the mandible to the mentum and from the occipital scalp along the sternocleidomastoid into the original plane of dissection. Defatting is carried out over and above the horizontal ramus of the mandible, feathering into the buccal region to achieve a smooth transition from the defatted platysma onto the nondefatted superficial musco-aponeurotic system (SMAS).

After the SMAS has been dealt with by either advancement or plication, additional submandibular and lateral platysma plication sutures may be placed to enhance further the neck contour.

\section{RESULTS}

Photographs (Figure 5, left and centre) show the profile of a 39-year-old woman with a fatty neck and flaccid platysma, before and after a conservative face lift, demonstrating no improvement of the neck. The same patient three years after a face and neck lift (Figure 5, right) with SMAS advancement, subcutaneous, subplatysmal and prehyoid neck lipectomy and corset platysmaplasty, shows sustained improvement of the neck profile. Patients' photographs (Figures 6,7,13-15) show the effectiveness of corset platysmaplasty in correcting prominent paramedian platysma bands and flaccid platysma muscle in a variety of clinical situations.

Corset platysmaplasty in conjunction with cervicofacial lipoplasty has been found to be indicated in the majority of my cervicofacial rhytidectomy procedures, and patients have been followed for up to nine years postoperatively. Although palpable plication ridges occasionally linger, they have not been visible and have disappeared within six months. Recurrent muscle bands have been rare and inconspicuous. Small submandibular gland bulges have been eliminated, and large bulges seen preoperatively have been improved by surgery rather than accentuated by surgery. (Several partial resections of large glands were performed at the time of platysmaplasty.)

Neck discomfort and a tight sensation was a complaint common to all platysmaplasty patients in this series and disappeared within 10 days of surgery. 

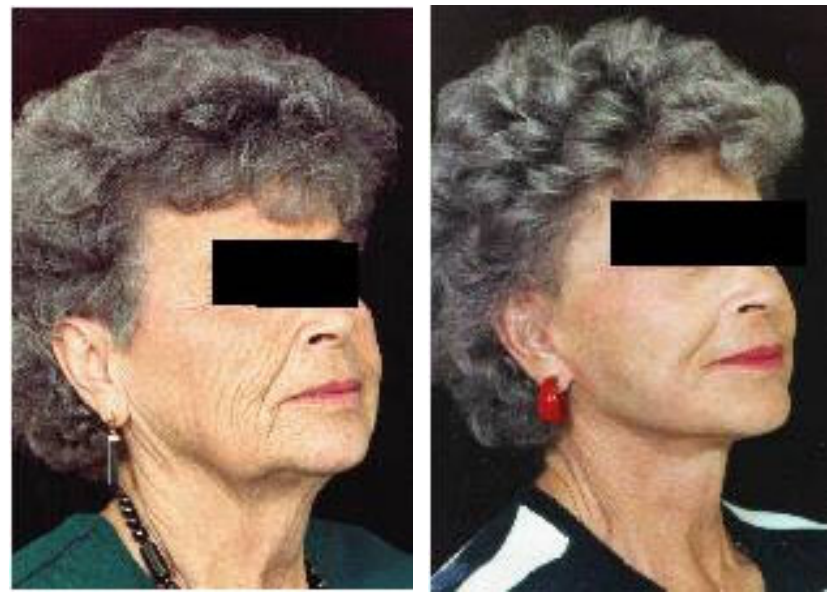

Figure 13) A 67-year-old woman shown before and one year after face and neck lift with jaw line and neck lipectomy and corset platysmaplasty
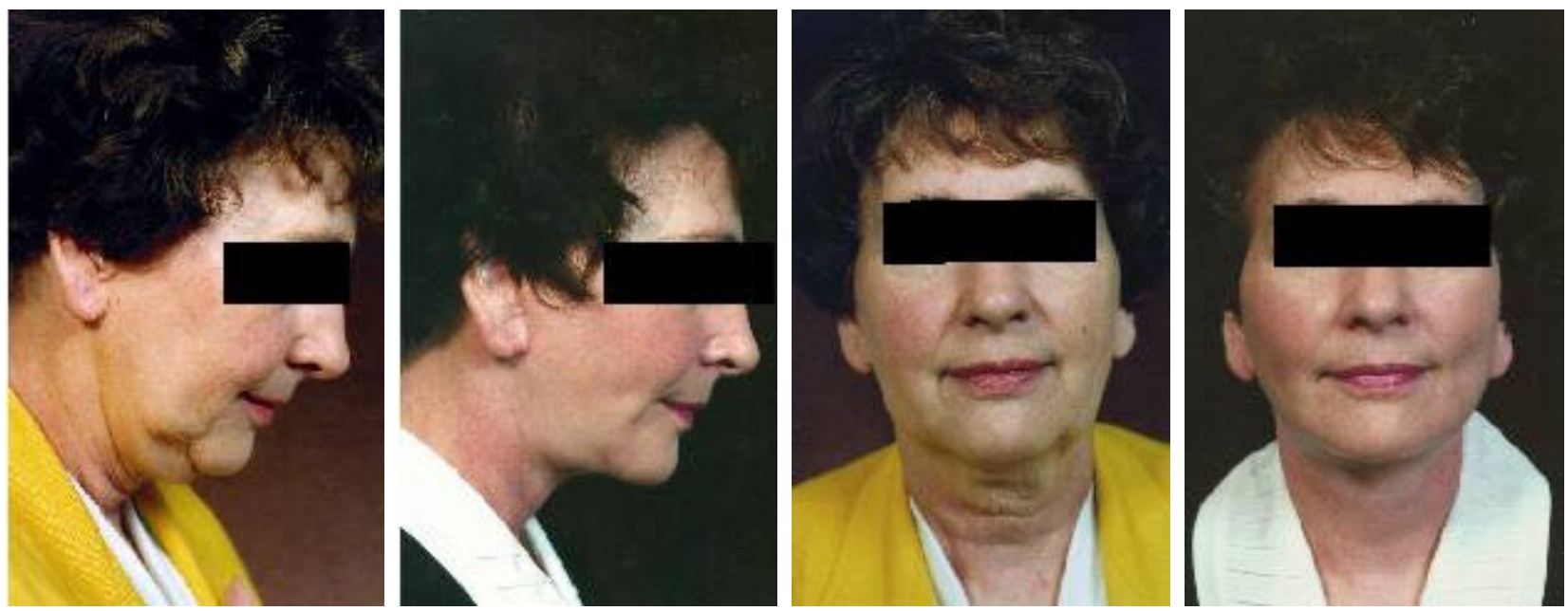

Figure 14) A 59-year-old woman with a heavy neck before and one year after face and neck lift with lower cheek lipectomy and superficial muscoaponeurotic system advancement advancement, subcutaneous and prehyoid neck lipectomy, and corset platysmaplasty 


\section{PERSONA USE ONIY - DO NOT COPY}

Whidden

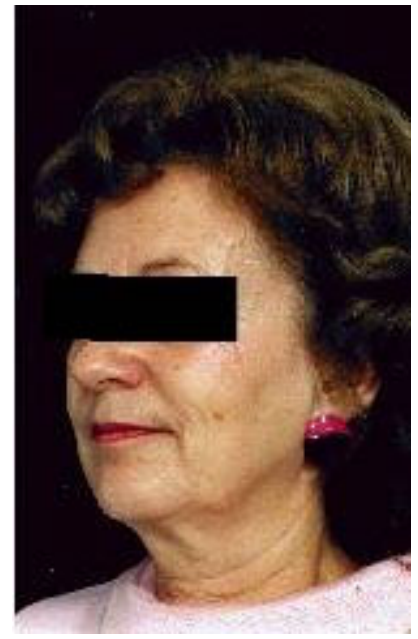

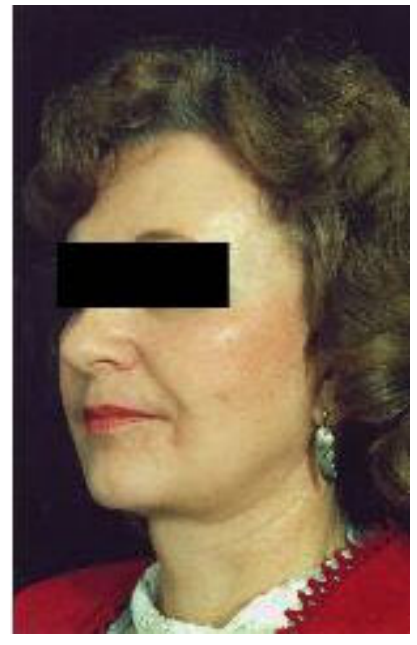
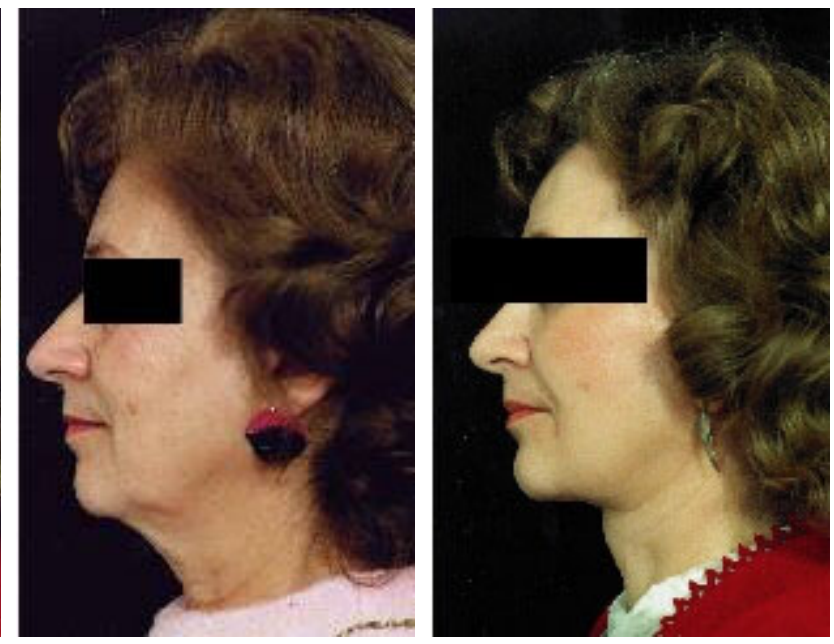

Figure 15) A 60-year-old woman with a fatty neck and flaccid platysma shown before and one year after coronal lift, four-lid blepharoplasty, circumoral dermabrasion, face and neck lift with superficial muscoaponeurotic system advancement advancement, subcutaneous, subplatysmal and prehyoid lipectomy and corset platysmaplasty

\section{DISCUSSION}

When treating the blunt cervicomental angle, whether excess subcutaneous central neck fat is present or not, central subplatysmal and prehyoid fat is usually found to be abundant at the time of surgery. Aggressive removal of prehyoid fat creates a sharplydefined cervicomental angle and facilities undermining of the medial platysmal borders and ease of advancement to the midline.

Two or three rows of continuous imbricating suturing of the newly relaxed medial platysmal layers creates a differential and progressive muscle-tightening sheet that shapes and conforms to the deeper neck contours to construct a smooth neck with a well-defined cervicomental angle.

\section{REFERENCES}

1. Ellenbogen R, Karlin JV. Visual criteria for success in restoring the youthful neck. Plast Recontr Surg 1980;66;826-37.

2. Aston SJ. Platysma-SMAS cervicofacial rhytidoplasty. Clin Plast Surg 1983;10:507520 .

3. Connell BF. Neck contour deformities. The art, engineering, anatomical diagnosis, architectural planning, and aesthetics of surgical correction. Clin Plast Surg 1987;14:68392.

4. Guerrerosantos J. Necklift: Simplified surgical technique, refinements, and clinical classification. Clin Plast Surg 1983;10:379-404.

5. Feldman JJ. Corset platysmaplasty. Plast Reconstr Surg 1990;85:333-343. 